\title{
Quantum Chemical Investigation of the Intramolecular Copigmentation Complex of an Acylated Anthocyanin
}

\author{
Juanjuan He ${ }^{a, \#}$ Xue Li, ${ }^{a, \#}$ Gustavo T. M. Silva, ${ }^{\oplus b}$ Frank H. Quina ${ }^{*, b}$ and \\ Adelia J. A. Aquino ${ }^{(1, a, c, d}$ \\ ${ }^{a}$ School of Pharmaceutical Science and Technology, Tianjin University, \\ 300072 Tianjin, People's Republic of China \\ ${ }^{b}$ Instituto de Química, Universidade de São Paulo, Av. Lineu Prestes 748, \\ 05508-030 São Paulo-SP, Brazil \\ 'Department of Chemistry and Biochemistry, Texas Tech University, \\ 79409-1061 Lubbock, Texas, United States \\ 'Institute for Soil Research, University of Natural Resources and Life Sciences Vienna, \\ Peter-Jordan-Strasse 82, A-1190 Vienna, Austria
}

\begin{abstract}
Anthocyanins are the natural plant pigments responsible for most of the red, blue and purple colors of flowers and fruit. One method of stabilization of the color of anthocyanins in nature is intramolecular copigmentation, in which a copigment molecule covalently attached to one of the sugar residues complexes with the anthocyanin cation chromophore. In the present work, two quantum chemical methodologies, time-dependent density functional theory (TD-DFT) and secondorder algebraic diagrammatic construction $(\mathrm{ADC}(2))$, were employed to predict the absorption spectra in vacuum and conductor-like screening model (COSMO) water of a natural anthocyanin containing an ester of coumaric acid (copigment) bound to the sugar residue of a cyanidin chromophore. $\mathrm{ADC}(2)$ in water adequately reproduces the experimental spectra with and without intramolecular copigmentation, pointing to this theoretical technique as a promising approach for predicting the spectroscopic properties of natural (and nature-inspired) dyes and pigments.
\end{abstract}

Keywords: anthocyanins, copigmentation, quantum chemistry, absorption spectra, ADC(2)

\section{Introduction}

Anthocyanins are the natural plant pigments responsible for most of the red, blue and purple colors of flowers and fruit. ${ }^{1-4}$ The thousands of different anthocyanins that have been described in the literature ${ }^{4,5}$ differ basically in the chemical nature of the sugars and other residues attached to one of six basic anthocyanidin cation chromophores (Scheme 1). In nature, the 7-hydroxy group is almost always free, the 3-hydroxy group is always glycosylated and the 5-hydroxy group of the anthocyanidin cation chromophore may or may not be glycosylated.

The following questions are of particular interest to us: (i) how do plants manipulate the structure and/or chemistry of the anthocyanins to produce such a broad palette of

*e-mail: quina@usp.br, adelia.aquino@tju.edu.cn, adelia.aquino@univie.ac.at

"These two authors contributed equally to this work. colors (all colors across the visible spectrum are known except green, for perhaps obvious reasons), ${ }^{6,7}$ (ii) what are the relationships between structure and stability, not just chemical, but also photochemical, in order to withstand large doses of solar radiation without fading $;{ }^{8-10}$ and (iii) how are color, structure and stability related to their biological roles in plants? ${ }^{8}$ A more profound understanding of these questions is central to the rational design of new anthocyanin-inspired dyes and pigments with desirable colors and stability and for the color stabilization of natural anthocyanins in practical applications such as cosmetics or foods. ${ }^{11-13}$

Although the maximum wavelength of the absorption, and hence the color of anthocyanidin cations shifts toward the red (longer wavelengths) with increasing number of hydroxy or methoxy substituents in the B-ring, ${ }^{5,6}$ this by itself cannot account for the much larger color changes observed in nature. At the same time, upon isolation from 


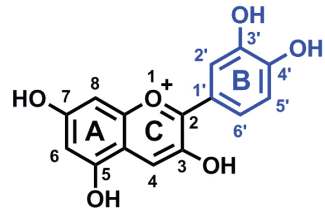

Cyanidin (50\%)

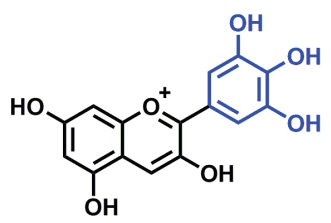

Delphinidin (12\%)

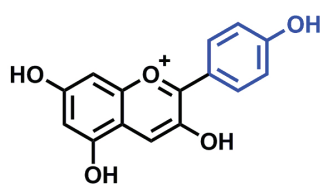

Pelargonidin (12\%)<smiles>COc1cc(-c2[o+]c3cc(O)cc(O)c3cc2O)ccc1O</smiles>

Peonidin (12\%)<smiles>COc1cc(-c2[o+]c3cc(O)cc(O)c3cc2O)cc(OC)c1O</smiles>

Malvidin (7\%)<smiles>COc1cc(-c2[o+]c3cc(O)cc(O)c3cc2O)cc(O)c1O</smiles>

Petunidin $\mathbf{( 7 \% )}$

Scheme 1. The six basic anthocyanin chromophoric units or anthocyanidins with the approximate relative percentages found in nature indicated in parentheses.

the plant, the color of natural anthocyanins is found to be quite sensitive to the $\mathrm{pH}$ of the medium. Anthocyanins are weak acids, with $\mathrm{p} K_{\mathrm{a}} \mathrm{s}$ for deprotonation of the 7-hydroxy group in the range of ca. $\mathrm{pH} 4-5 .{ }^{7}$ Although the conjugate bases (A) absorb at slightly longer wavelengths and are more bluish in color, this is of limited utility for changing the color due to competitive $\mathrm{pH}$-dependent chemical equilibria that transform the colored anthocyanin cation $\left(\mathrm{AH}^{+}\right)$into colorless or near-colorless products above about $\mathrm{pH}$ 3. Thus, upon increasing the $\mathrm{pH}$ of an aqueous solution of an anthocyanin to neutral $\mathrm{pH}$, the cation $\left(\mathrm{AH}^{+}\right)$ undergoes hydration (time scale of seconds) to form the hemiketal (B), followed by ring-opening tautomerism (time scale of minutes) to the $(E)$-chalcone (EC), which in turn can isomerize (time scale of hours) to the $(Z)$-chalcone (ZC). Acidification to $\mathrm{pH}$ 1-2 reverts the system back to the cation form $\mathrm{AH}^{+}$, recovering the color. ${ }^{8}$

One obvious way to prevent the hydration-dependent loss of color of anthocyanins would be to isolate or encapsulate the anthocyanin in an environment that prevents contact with water. Indeed, inclusion of the 3',4',7-trimethoxyflavylium cation, a synthetic analog of the anthocyanin chromophore, in the cavity formed by two molecules of cucurbit[7]uril does stabilize its color up to near neutral $\mathrm{pH} \cdot{ }^{14}$ Likewise, incorporation of anthocyanins into anionic micelles of sodium dodecyl sulfate (SDS) also stabilizes the color to higher solution $\mathrm{pH}$ due to the higher local concentration of protons at the micelle surface combined with additional electrostatic stabilization of the cationic form $\left(\mathrm{AH}^{+}\right)$relative to the neutral forms (A, B, EC and ZC). ${ }^{15}$

A second mechanism that contributes to color stabilization in plants is copigmentation. ${ }^{10,16-23}$ In the plant vacuoles where anthocyanins concentrate, ${ }^{24-26}$ the anthocyanin cation $\mathrm{AH}^{+}$can chelate with metal cations ${ }^{23,27}$ such as $\mathrm{Al}^{3+}$ and/or form complexes with colorless organic copigment molecules, such as electron-rich derivatives of hydroxybenzoic or hydroxycinnamic acids, flavones or one of the colorless neutral forms of the anthocyanin itself. 10,16-23 Metal cation chelation can lead to large changes in the color, primarily from red to blue as, for example, in Hydrangea ${ }^{28}$ but is limited to anthocyanins with two or more free $\mathrm{OH}$ groups in the B-ring ${ }^{23,27,29}$ (i.e., anthocyanins derived from cyanidin, delphinidin and petunidin). In contrast, copigmentation via complexation with organic molecules results in much smaller red shifts of the absorption, but can increase the $\mathrm{pH}$ at which hydration occurs, consistent with steric hindrance to attack of water and charge transfer from the copigment to the anthocyanin as an important contributor to the stability of the anthocyanin-copigment complex. ${ }^{10,16-23}$ One limitation of this bimolecular copigmentation is the range of stability constants for the complexation, ${ }^{21,23}$ which rarely exceed $10^{4} \mathrm{M}^{-1}$, requiring $\mathrm{mM}$ or greater local copigment concentrations in order to achieve a substantial percentage of complexation of the anthocyanin. Some plants have overcome the entropic limitations of bimolecular copigmentation by covalently attaching one or more copigment molecules to the sugar residues of the anthocyanin (e.g., acyl ester derivatives), ${ }^{18-20,22,23}$ transforming the copigmentation into an entropically much more favorable intramolecular complexation phenomenon.

The photochemical stability of anthocyanins is the result of the existence of several extremely efficient, ultrafast pathways for the transformation of absorbed light energy into heat. The lowest excited singlet state of anthocyanins is a much stronger acid than the ground state, with excited state $\mathrm{p} K_{\mathrm{a}}{ }^{*} \mathrm{~s}$ in the range of -1 to 0 . Thus, upon electronic excitation of an anthocyanin cation in $\mathrm{pH} 2$ aqueous solution, the lowest excited singlet state of the cation $\left(\mathrm{AH}^{+*}\right)$ undergoes ultra-rapid adiabatic proton transfer to water (in ca. 5-20 ps) to produce the excited singlet state of the conjugate base $\mathrm{A}^{*}$, which in turn decays rapidly to 
its ground state (in ca. 200 ps), where reprotonation returns the system to its pre-excitation state. ${ }^{8,9}$ The excited states of copigmentation complexes are deactivated by an additional even more efficient pathway, presumably charge-transfermediated internal conversion, which funnels the singlet excited state of the complex back to the ground state in less than a ps. ${ }^{10,22}$

During eons of evolution, the interactions of plants with animals and their environment have resulted in the appearance of pigments that attract (or perhaps warn away) pollinators, frugivores or herbivores or serve as excess solar radiation filters. ${ }^{30-33}$ In practical terms, a solid theoretical understanding of the relationship between the structure, spectroscopy and photochemistry of natural plant pigments has important implications for their use as safe dyes or coloring agents in foods and pharmaceutical preparations, where long-term stability and lightfastness of the color are important parameters. In the present work, we compare the ability of two quantum chemical methodologies to predict the effects of intramolecular copigmentation on the absorption spectra of an acyl anthocyanin. Comparison with experimental data ${ }^{22}$ for this anthocyanin with and without the acyl copigments chromophore provides clear guidelines for further work envisioning the rational computational design and development of new classes of synthetic natureinspired dyes and pigments for a variety of applications in consumer products.

\section{Methodology}

\section{Quantum chemical calculations}

The geometry optimization and spectroscopic analyses were performed with a standard Becke, 3-parameter, Lee-Yang-Parr (B3-LYP $)^{34}$ and the triple zeta valence polarization (TZVP) ${ }^{35}$ basis set. Generalized polar solvent effects were included by employing the conductor-like screening model (COSMO) ${ }^{36,37}$ to mimic water during the geometry optimization procedure. All calculations were performed using the Turbomole ${ }^{38}$ suite of programs. The calculations of the vertical electronic states were performed by means of time-dependent density functional theory (TD-DFT) $)^{39,40}$ and by the second-order algebraic diagrammatic construction $(\operatorname{ADC}(2))^{41}$ with TZVP basis set. The $\mathrm{ADC}(2)$ method is combined with the resolution of identity (RI), ${ }^{42}$ allowing an efficient handling of the twoelectron integrals. The energies of the five lowest excited states were calculated in both vacuum and taking water as polar environment based on the B3-LYP/TZVP optimized geometries in vacuum and, in the latter case, the geometries reoptimized including the effect of the polar medium.

\section{Calculated absorption spectra}

The band shapes of the theoretically predicted absorption spectra were constructed in Microsoft Excel from the vertical excitation energies $\left(E_{i}\right.$, in $\left.e V\right)$ and the corresponding oscillator strengths $\left(f_{i}\right)$ for the first 5 excited states as a sum of superimposed Gaussian functions centered on each $\mathrm{E}_{\mathrm{i}} \cdot{ }^{43}$

$\varepsilon(\bar{v})=26954 \sum_{\mathrm{i}}\left(\frac{f_{i}}{\Delta_{1 / 2}}\right) \exp \left[-2.733\left(\frac{\mathrm{E}-\mathrm{E}_{\mathrm{i}}}{\Delta_{1 / 2}}\right)^{2}\right]$

where $\Delta_{1 / 2}$ is the spectral bandwidth (full width at half maximum) in $\mathrm{eV}$ and $\varepsilon(\bar{v})$ is the molar absorption coefficient (in $\mathrm{M}^{-1} \mathrm{~cm}^{-1}$ ). A spectral bandwidth of $0.36 \mathrm{eV}$, an average value determined for the long-wavelength absorption band of several experimental absorption spectra of anthocyanins, was used for all of the electronic transitions. Spectra were plotted as $\varepsilon(\lambda)$ versus wavelength $(\lambda$, in $\mathrm{nm})$ and normalized to permit comparison with the experimental spectra. The published absorption spectra ${ }^{22}$ were digitized with the online application WebPlot Digitizer. ${ }^{44}$

\section{Results and Discussion}

The acyl anthocyanin CyCoum (cyanidin $3-O-\beta-(6 "-O-E-p$ - coumaroyl-s a mbubioside $)$ $5-O$ - $\beta$-glucopyranoside) chosen for this study is a natural acylated anthocyanin, sold commercially by Polyphenols ${ }^{45}$ and found, e.g., in elderberries and other fruits and flowers. CyCoum consists of a $p$-coumaric acid (Coum) residue covalently attached via an ester linkage to a hydroxyl group of one of the 3-O-sugar residues of a cyanidin3,5-O-diglycoside ( $\mathrm{Cy}$ ) chromophore (Scheme 2). In acidic (pH 1) aqueous solution, CyCoum exhibits two significant absorption maxima above $300 \mathrm{~nm}$, a band at $314 \mathrm{~nm}$ (molar absorption coefficient of $\varepsilon=16000 \mathrm{M}^{-1} \mathrm{~cm}^{-1}$ ), due to the presence of the Coum ester chromophore, and a band at $520 \mathrm{~nm}\left(\varepsilon=26000 \mathrm{M}^{-1} \mathrm{~cm}^{-1}\right){ }^{22}$ The $520 \mathrm{~nm}$ band is shifted about $12 \mathrm{~nm}$ to the red and decreases in intensity relative to the long wavelength absorption band of $\mathrm{Cy}$ (508 nm, $\left.\varepsilon=32000 \mathrm{M}^{-1} \mathrm{~cm}^{-1}\right){ }^{22}$ Both $\mathrm{Cy}$ and CyCoum have short ca. 4-5 ps fluorescence lifetimes due to fast excited state proton transfer to water, but $\mathrm{Cy}$ has a 2.2-fold higher fluorescence quantum yield, consistent with partial intramolecular copigmentation (ca. 60-65\%) in CyCoum. Further evidence of the presence of an intramolecular copigmentation complex in CyCoum is provided by the decay of the CyCoum excited singlet state, which indicates a competition between excited state proton transfer and an 
additional ultrafast internal conversion pathway back to the ground state, and the partial protection against attack of water ( 0.4 unit increase in the $\mathrm{p} K$ value for hydration) in CyCoum relative to $\mathrm{Cy}_{.}{ }^{22}$
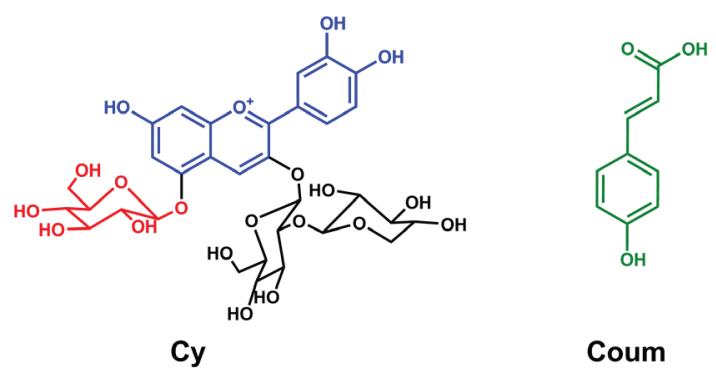

CyCoum:

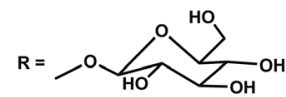

5-MeO-CyCoum:

$\mathrm{R}=-\mathrm{OCH}_{3}$

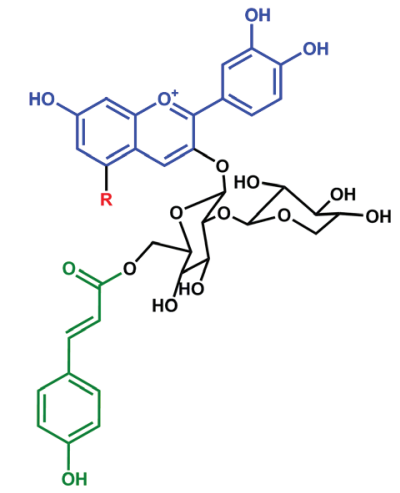

Scheme 2. Structures of the cyanine derivative without the attached copigment (Cy), coumaric acid (Coum), the 5-O-glucosyl acyl cyanine derivative with the covalently attached copigment (CyCoum) and its 5-methoxy analog (5-MeO-CyCoum).

Geometry optimizations were initially performed in the gas phase for cyanidin-3,5-O-diglucoside (Cy) and the glycosylated acyl anthocyanin CyCoum with the covalently attached Coum-ester copigments chromophore by the B3-LYP/TZVP approach in vacuum. Although the optimized structure of the former was deemed satisfactory, the final geometry of CyCoum indicated a rather severe restriction to the mobility of Coum-ester group relative to the cyanidin chromophore due to multiple hydrogen bonds between the 3-O- and 5-O-sugar residues (Figure 1), that would presumably be of much less importance in aqueous solution due to hydrogen bonding to water. Because the addition of up to five additional water molecules hydrogen-bonded to selected $\mathrm{OH}$ groups of the molecule failed to resolve fully the conformational restrictions, the 5-O-sugar residue was replaced with a methoxy group (5-MeO-CyCoum) so that the conformational space of the 3-O-sugar and the attached Coum-ester chromophore could be appropriately explored during the geometry optimization. As shown in Figure 1, the Coum-ester chromophore of 5-MeO-CyCoum lies in relatively intimate contact with the cyanidin chromophore, maximizing the interchromophoric interaction.
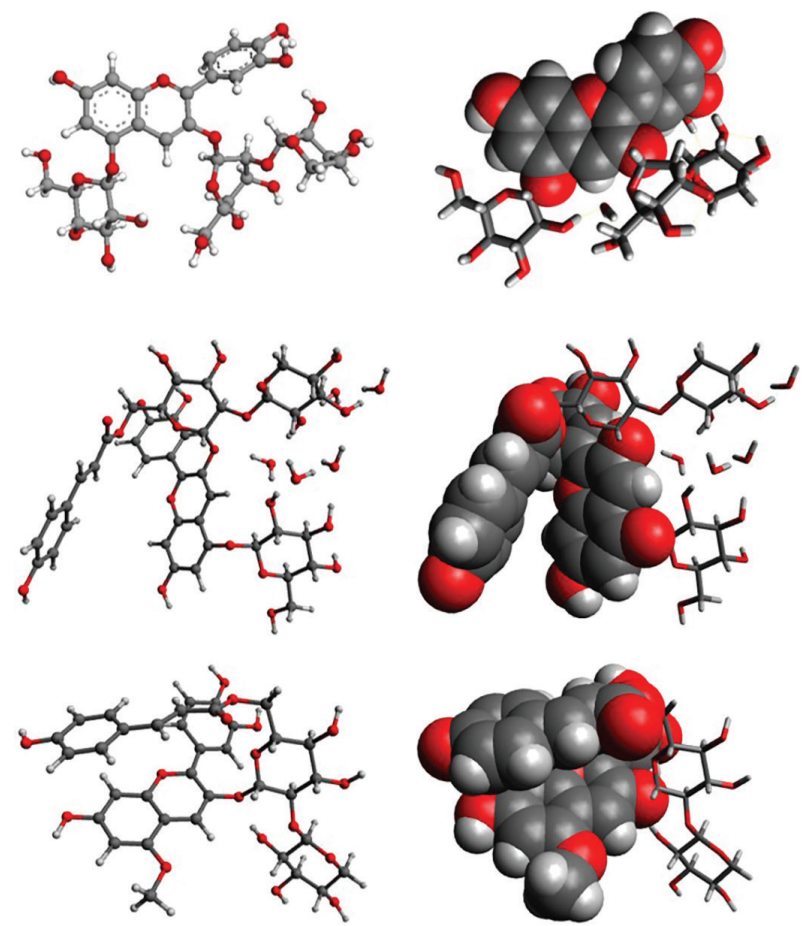

Figure 1. Minimized conformations of Cy (upper), CyCoum (middle) and 5-MeO-CyCoum (lower).

Excitation energies $(\Delta \mathrm{E})$, corresponding maximum wavelengths $(\lambda)$ and oscillator strengths $(f)$ for the 5 lowest excited states of Cy calculated by TD-DFT in vacuum and in water (COSMO) employing the B3-LYP/TZVP geometries optimized in vacuum or water, respectively, are listed in Table 1, and the corresponding predicted spectra illustrated in Figure 2a. The analogous results for Cy calculated at the ADC(2)/TZVP level of theory are shown in Table 2 and Figure $2 b$ for comparison. Three important conclusions emerge from these results: (i) the differences in energies of the upper excited states and the oscillator strengths are relatively insensitive to the difference between geometry optimizations in vacuum and in water; (ii) the most notable difference is a slight increase in the energy of state $S_{1}$ in water at both levels of theory; (iii) the $\mathrm{S}_{0} \rightarrow \mathrm{S}_{1}$ absorption maxima predicted by the ADC(2)/TZVP approach are in better agreement with the experimental value, though still too high by almost $20 \mathrm{~nm}$ in a polar environment (water); and (iv) the $\mathrm{S}_{0} \rightarrow \mathrm{S}_{3}$ oscillator strength appears to be overestimated to a greater extent in the TD-DFT approach.

Tables 3 and 4 compare the computational results for the first 5 excited states of CyCoum, which has the less-than-optimal interaction between the anthocyanin and coumaric ester chromophores, and 5-MeO-CyCoum, where the conformational restriction imposed by hydrogen bonding in the sugar residues was relaxed. Here there are clear differences between the lowest two excited states at 
Table 1. Excitation energies $(\Delta \mathrm{E})$, maximum wavelengths $(\lambda)$ and oscillator strengths for the 5 lowest excited states of Cy by the B3-LYP/TZVP approach in vacuum and in water using geometries optimized in vacuum or in water, respectively

\begin{tabular}{|c|c|c|c|c|c|c|}
\hline \multirow{2}{*}{ Excited state } & \multicolumn{3}{|c|}{ B3-LYP/TZVP/vacuum } & \multicolumn{3}{|c|}{ B3-LYP/TZVP/COSMO } \\
\hline & $\Delta \mathrm{E} / \mathrm{eV}$ & $\lambda / \mathrm{nm}$ & Oscillator strength & $\Delta \mathrm{E} / \mathrm{eV}$ & $\lambda / \mathrm{nm}$ & Oscillator strength \\
\hline 1 & 2.58 & 481 & 0.569 & 2.61 & 475 & 0.560 \\
\hline 2 & 2.92 & 425 & 0.045 & 2.91 & 426 & 0.035 \\
\hline 3 & 3.29 & 377 & 0.149 & 3.29 & 377 & 0.212 \\
\hline 4 & 3.50 & 354 & 0.013 & 3.50 & 354 & 0.0044 \\
\hline 5 & 3.51 & 353 & 0.0003 & 3.64 & 341 & 0.0069 \\
\hline
\end{tabular}

B3-LYP: Becke, 3-parameter, Lee-Yang-Parr; TZVP: triple zeta valence polarization; COSMO: conductor-like screening model.

Table 2. Excitation energies $(\Delta \mathrm{E})$, maximum wavelengths $(\lambda)$ and oscillator strengths for the 5 lowest excited states of Cy by the ADC $(2) / T Z V P$ approach in vacuum and in water using geometries optimized in vacuum or in water, respectively

\begin{tabular}{|c|c|c|c|c|c|c|}
\hline \multirow{2}{*}{ Excited state } & \multicolumn{3}{|c|}{$\mathrm{ADC}(2) / \mathrm{TZVP} /$ vacuum } & \multicolumn{3}{|c|}{$\mathrm{ADC}(2) / \mathrm{TZVP} / \mathrm{COSMO}$} \\
\hline & $\Delta \mathrm{E} / \mathrm{eV}$ & $\lambda / \mathrm{nm}$ & Oscillator strength & $\Delta \mathrm{E} / \mathrm{eV}$ & $\lambda / \mathrm{nm}$ & Oscillator strength \\
\hline 1 & 2.47 & 502 & 0.664 & 2.53 & 490 & 0.664 \\
\hline 2 & 3.02 & 411 & 0.0066 & 3.02 & 411 & 0.011 \\
\hline 3 & 3.53 & 351 & 0.097 & 3.29 & 349 & 0.102 \\
\hline 4 & 4.05 & 306 & 0.052 & 3.50 & 303 & 0.056 \\
\hline 5 & 4.20 & 295 & 0.0027 & 3.64 & 287 & 0.0016 \\
\hline
\end{tabular}

ADC(2): second-order algebraic diagrammatic construction; TZVP: triple zeta valence polarization; COSMO: conductor-like screening model.
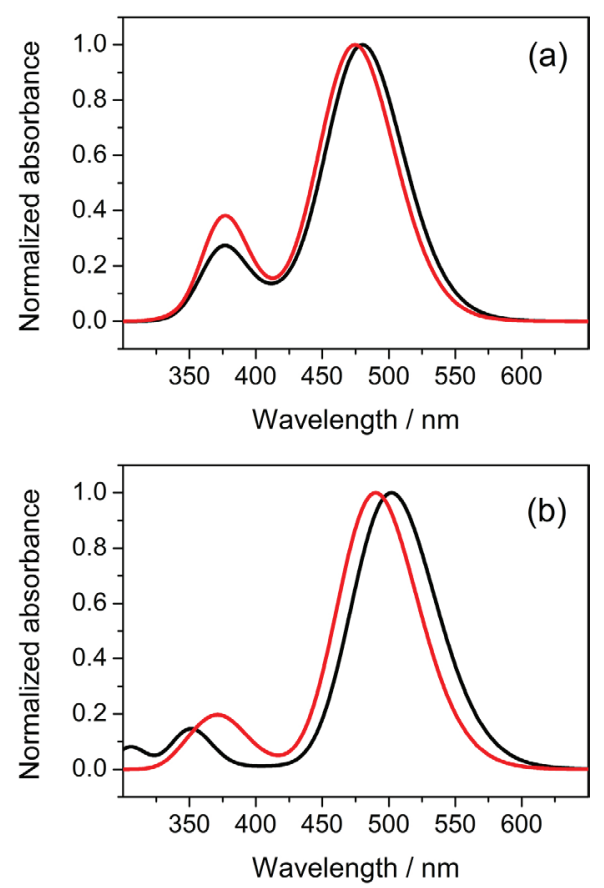

Figure 2. Predicted absorption spectra of Cy in vacuum (black) and in COSMO water (red) by: (a) TD-DFT/B3-LYP/TZVP (from data in Table 1); (b) ADC(2)/TZVP (from data in Table 2).

the ADC(2)/TZVP level and the lowest three states at the TD-DFT level of theory, probably reflecting the known limitations of TD-DFT with the conventional B3-LYP functional in the treatment of excited states that, as in the present case, ${ }^{22}$ have appreciable charge-transfer character (a problem that can in principle be ameliorated by the use of a range-separated hybrid) ${ }^{40}$ Note in particular that the excited state energies of the conformation adopted by this restricted conformation of CyCoum are much closer to those of Cy than to those of 5-MeO-CyCoum, consistent with the anticipated short-range, overlap-dependent nature of the interaction between the anthocyanin and copigment chromophores in copigmentation complexes.

Figure 3 compares the absorption spectra of $\mathrm{Cy}$ and 5-MeO-CyCoum (normalized relative to $\mathrm{Cy}$ ) predicted by ADC(2)/TZVP in water with the corresponding experimental spectra of $\mathrm{Cy}$ and CyCoum in aqueous solution. The calculated spectra nicely reproduce the two most salient features of the experimental spectra, i.e., the slight shift of the long-wavelength band of the spectrum to the red, together with a moderate decrease in its intensity that appears to be characteristic of copigmentation of this type. Moreover, when the spectrum of CyCoum is simulated as 35:65 percent ratio of the $\mathrm{Cy}$ and 5-MeO-CyCoum spectra, consistent with the approximate extent of intramolecular copigmentation, an even closer match to the relative intensities observed in the experimental spectra is obtained.

\section{Conclusions}

In summary, compared to TD-DFT/B3-LYP/TZVP, the ADC(2)/TZVP approach provides a much superior description of the excited state energies and of the optical absorption spectrum of this system. In particular, the absorption spectra predicted by ADC(2)/TZVP in a polar 
Table 3. Excitation energies $(\Delta \mathrm{E})$, maximum wavelengths $(\lambda)$ and oscillator strengths for the 5 lowest excited states of 5-glu-CyCoum by TD-DFT/TZVP and ADC(2)/TZVP approaches in water using the B3-LYP/TZVP geometry optimized in water

\begin{tabular}{|c|c|c|c|c|c|c|}
\hline \multirow{2}{*}{ Excited state } & \multicolumn{3}{|c|}{ TD-DFT/TZVP/COSMO } & \multicolumn{3}{|c|}{ ADC(2)/TZVP/COSMO } \\
\hline & $\Delta \mathrm{E} / \mathrm{eV}$ & $\lambda / \mathrm{nm}$ & Oscillator strength & $\Delta \mathrm{E} / \mathrm{eV}$ & $\lambda / \mathrm{nm}$ & Oscillator strength \\
\hline 1 & 2.28 & 544 & 0.000 & 2.52 & 492 & 0.566 \\
\hline 2 & 2.51 & 494 & 0.311 & 3.05 & 407 & 0.046 \\
\hline 3 & 2.91 & 426 & 0.304 & 3.41 & 364 & 0.050 \\
\hline 4 & 3.26 & 380 & 0.046 & 3.69 & 336 & 0.0001 \\
\hline 5 & 3.34 & 371 & 0.002 & 4.04 & 307 & 0.056 \\
\hline
\end{tabular}

TD-DFT: time-dependent density functional theory; TZVP: triple zeta valence polarization; COSMO: conductor-like screening model; ADC(2): secondorder algebraic diagrammatic construction.

Table 4. Excitation energies $(\triangle \mathrm{E})$, maximum wavelengths $(\lambda)$ and oscillator strengths for the 5 lowest excited states of 5-MeO-CyCoum by TD-DFT/ TZVP and ADC(2)/TZVP approaches in water using the B3-LYP/TZVP geometry optimized in vacuum

\begin{tabular}{|c|c|c|c|c|c|c|}
\hline \multirow{2}{*}{ Excited state } & \multicolumn{3}{|c|}{ TD-DFT/TZVP/COSMO } & \multicolumn{3}{|c|}{$\mathrm{ADC}(2) / \mathrm{TZVP} / \mathrm{COSMO}$} \\
\hline & $\Delta \mathrm{E} / \mathrm{eV}$ & $\lambda / \mathrm{nm}$ & Oscillator strength & $\Delta \mathrm{E} / \mathrm{eV}$ & $\lambda / \mathrm{nm}$ & Oscillator strength \\
\hline 1 & 2.27 & 547 & 0.014 & 2.42 & 512 & 0.467 \\
\hline 2 & 2.55 & 486 & 0.380 & 2.86 & 433 & 0.060 \\
\hline 3 & 2.74 & 452 & 0.004 & 3.20 & 388 & 0.005 \\
\hline 4 & 3.21 & 386 & 0.244 & 3.42 & 363 & 0.103 \\
\hline 5 & 3.41 & 364 & 0.001 & 4.03 & 308 & 0.057 \\
\hline
\end{tabular}

TD-DFT: time-dependent density functional theory; TZVP: triple zeta valence polarization; COSMO: conductor-like screening model; ADC(2): secondorder algebraic diagrammatic construction.
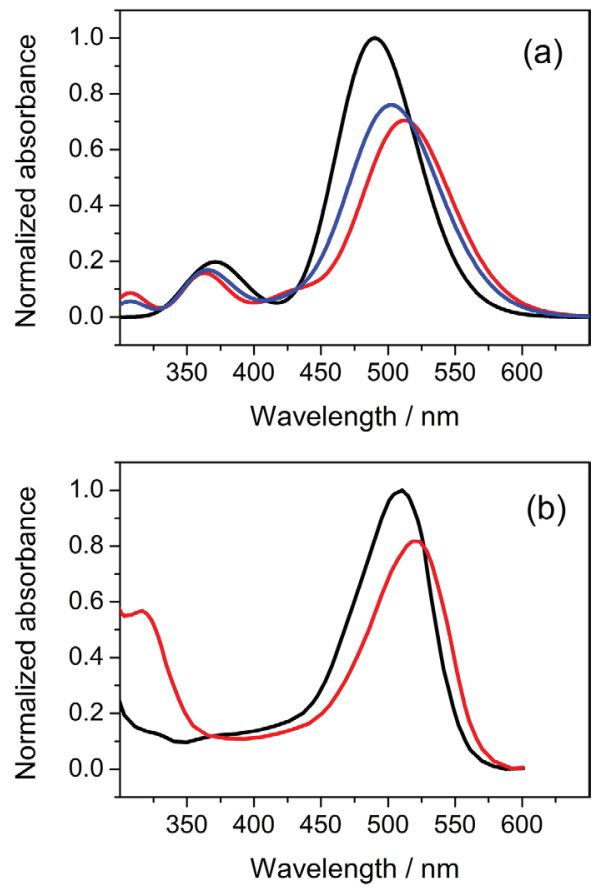

Figure 3. Comparison of the absorption spectra of: (a) Cy (black), 5-MeO-CyCoum (red) and a 35:65 proportion of Cy:5-MeO-CyCoum (blue), normalized relative to Cy, predicted by ADC(2)/TZVP/COSMO, with (b) the normalized experimental absorption spectra of Cy (as the 3,5-diglucoside, black) and CyCoum (red). ${ }^{22}$

environment (water) nicely reproduce the two characteristic spectroscopic effects induced by copigmentation, the slight red shift and reduction in molar absorptivity of the long-wavelength absorption band. The combination of B3-LYP/TZVP geometry optimization with ADC(2)/ TZVP and COSMO program to take into account solvent effects thus appears to constitute a promising ensemble of theoretical techniques for obtaining a deeper understanding of the spectroscopic properties of both natural (and natureinspired) dyes and pigments.

\section{Acknowledgments}

Generous support by the School of Pharmaceutical Science and Technology (SPST), Tianjin University, China, including computer time on the Arran computer cluster at SPST, is gratefully acknowledged. F. H. Q. and G. T. M. S. thank the CNPq and CAPES, Brazil, for fellowships and NAP-PhotoTech, INCT-Catálise (CNPq 465454/2014-3), and CNPq (FHQ Universal grant 408181/2016-3) for funding. This work was carried out under the auspices of the SPST-TJU/IQ-USP cooperative exchange agreement. This study was financed in part by the Coordenação de Aperfeiçoamento de Pessoal de Nível Superior (CAPES, Brazil), Finance Code 001.

\section{References}

1. Castaneda-Ovando, A.; Pacheco-Hernandez, M. L.; PaezHernandez, M. E.; Rodriguez, J. Á.; Galan-Vidal, C. A.; Food Chem. 2009, 113, 859. 
2. Pina, F.; Melo, M. J.; Laia, C. A. T.; Parola, A. J.; Lima, J. C.; Chem. Soc. Rev. 2012, 41, 869.

3. Pina, F.; Oliveira, J.; Freitas, V.; Tetrahedron 2015, 71, 3107.

4. Quina, F. H.; Bastos, E. L.; An. Acad. Bras. Cienc. 2018, 90, 681.

5. Cabrita, L.; Fossen, T. T.; Andersen, Ø. M.; Food Chem. 2000, 68, 101.

6. Freitas, A. A.; Shimizu, K.; Dias, L. G.; Quina, F. H.; J. Braz. Chem. Soc. 2007, 18, 1537.

7. Freitas, A. A.; Dias, L. G.; Maçanita, A. A. L.; Quina, F. H.; J. Phys. Org. Chem. 2011, 24, 1201.

8. Quina, F. H.; Moreira, P. F.; Vautier-Giongo, C.; Rettori, D.; Rodrigues, R. E.; Freitas, A. A.; Silva, P. F.; Maçanita, A. L.; Pure Appl. Chem. 2009, 81, 1687.

9. Silva, V. O.; Freitas, A. A.; Maçanita, A. L.; Quina, F. H.; J. Phys. Org. Chem. 2016, 29, 594.

10. Rodrigues, R. F.; Silva, P. F.; Shimizu, K.; Freitas, A. A.; Kovalenko, S. A.; Ernsting, N. P.; Quina, F. H.; Maçanita, A. L.; Chem. - Eur. J. 2009, 15, 1397.

11. Cortez, R.; Luna-Vital, D. A.; Margulis, D.; Gonzalez de Mejia, E.; Compr. Rev. Food Sci. Food Saf. 2017, 16, 180.

12. Santos-Buelga, C.; Mateus, N.; Freitas, V.; J. Agric. Food Chem. 2014, 62, 6879.

13. Sigurdson, G. T.; Tang, P.; Giusti, M. M.; Annu. Rev. Food Sci. Technol. 2017, 8, 261.

14. Held, B.; Tang, H.; Natarajan, P.; da Silva, C. P.; Silva, V. O.; Bohne, C.; Quina, F. H.; Photochem. Photobiol. Sci. 2016, 15, 752.

15. Lima, J. C.; Vautier-Giongo, C.; Lopes, A.; Melo, E.; Quina, F. H.; Maçanita, A. L.; J. Phys. Chem. A 2002, 106, 5851.

16. Di Meo, F.; Sancho Garcia, J. C.; Dangles, O.; Trouillas, P.; J. Chem. Theory Comput. 2012, 8, 2034.

17. Sigurdson, G. T.; Tang, P.; Giusti, M. M.; Molecules 2018, 23, 598.

18. Yoshida, K.; Oyama, K.-i.; Kondo, T. In Recent Advances in Polyphenol Research; Yoshida, K.; Cheynier, V.; Quideau, S., eds.; John Wiley \& Sons, Ltd.: London, 2017, ch. 8, p. 171-192.

19. Mendoza, J.; Basílio, N.; Pina, F.; Kondo, T.; Yoshida, K.; J. Phys. Chem. B 2018, 122, 4982.

20. Moloney, M.; Robbins, R. J.; Collins, T. M.; Kondo, T.; Yoshida, K.; Dangles, O.; Dyes Pigm. 2018, 158, 342.

21. Silva, P. F.; Lima, J. C.; Freitas, A. A.; Shimizu, K.; Maçanita, A. L.; Quina, F. H.; J. Phys. Chem. A 2005, 109, 7329.

22. Silva, P. F.; Paulo, L.; Barbafina, A.; Elisei, F.; Quina, F. H.; Maçanita, A. L.; Chem. - Eur. J. 2012, 18, 3736.

23. Trouillas, P.; Sancho-García, J. C.; Freitas, V.; Gierschner, J.; Otyepka, M.; Dangles, O.; Chem. Rev. 2016, 116, 4937.
24. Pourcela, L.; Irania, N. G.; Lua, Y.; Riedl, K.; Schwartz, S.; Grotewold, E.; Mol. Plant 2010, 3, 78.

25. Raghvendra; Sharma, V.; Shakya, A.; Hedaytullah, M. D.; Arya, G. S.; Mishra, A.; Gupta, A. D.; Pachpute, A. P.; Patel, D.; Int. J. Pharm. Sci. Rev. Res. 2011, 6, 28.

26. Faraco, M.; Spelt, C.; Bliek, M.; Verweij, W.; Hoshino, A.; Espen, L.; Prinsi, B.; Jaarsma, R.; Tarhan, E.; de Boer, A. H.; Di Sansebastiano, G.-P.; Koes, R.; Quattrocchio, F. M.; Cell Rep. 2014, 6, 32.

27. Yoshida, K.; Mori, M.; Kondo, T.; Nat. Prod. Rep. 2009, 26, 884.

28. Ito, T.; Oyama, K.-i.; Yoshida, K.; Molecules 2018, 23, 1424.

29. Mora-Soumille, N.; Al Bittar, S.; Rosa, M.; Dangles, O.; Dyes Pigm. 2013, 96, 7.

30. Gould, K. S.; Lee, D. W.; Advances in Botanical Research, vol. 37; Gould, K.; Lee, D., eds.; Academic Press: New York, 2002.

31. Miller, R.; Owens, S. J.; Rørslett, B.; Opt. Laser Technol. 2011, 43, 282.

32. Stournaras, K. E.; Lo, E.; Böhning-Gaese, K.; Cazetta, E.; Dehling, D. M.; Schleuning, M.; Stoddard, M. C.; Donoghue, M. J.; Prum, R. O.; Schaefer, H. M.; New Phytol. 2013, 198, 617.

33. Landi, M.; Tattini, M.; Gould, K. S.; Environ. Exp. Bot. 2015, $119,4$.

34. Becke, A. D.; Phys. Rev. A 1988, 38, 3098.

35. Schäfer, A.; Huber, C.; Ahlrichs, R.; J. Chem. Phys. 1994, 100, 5829.

36. Klamt, A.; Schüürmann, G.; J. Chem. Soc., Perkin Trans. 2 1993, 799.

37. Klamt, A.; Jonas, V.; J. Chem. Phys. 1996, 105, 9972.

38. Ahlrichs, R.; Bär, M.; Häser, M.; Horn, H.; Kölmel, C.; Chem. Phys. Lett. 1989, 162, 165.

39. Dreuw, A.; Weisman, J. L.; Head-Gordon, M.; J. Chem. Phys. 2003, 119, 2943.

40. Adamo, C.; Jacquemin, D.; Chem. Soc. Rev. 2013, 42, 845.

41. Schirmer, J.; Phys. Rev. A 1982, 26, 2395.

42. Hättig, C.; Weigend, F. J.; J. Chem. Phys. 2000, 113, 5154.

43. Gorelsky, S. I.; Lever, A. B. P.; J. Organomet. Chem. 2001, 635, 187.

44. https://automeris.io/WebPlotDigitizer/index.html, accessed on July 17, 2018.

45. http://polyphenols.com/cyanidin-products/cyanidin-3coumsamb-5-glucoside-article145-186.html, accessed on July $17,2018$.

Submitted: July 31, 2018

Published online: November 29, 2018 